\title{
L'ENTRETIEN
}

\author{
De Mélanie Doyon et Juan-Luis Klein avec
}

\section{Vincent van Schendel \\ Directeur général de l'organisme \\ Territoires innovants en économie sociale et solidaire (TIESS)}

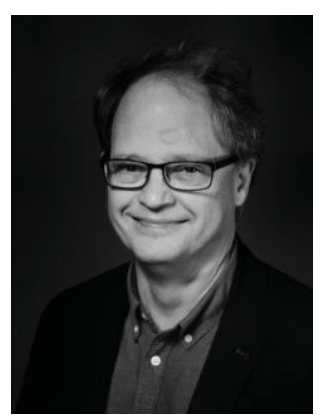

Photo : Sylvain Laroche

Détenteur d'une maitrise en économie de l'UQAM et d'un doctorat en relations industrielles de l'Université Laval, Vincent van Schendel œuvre depuis plus de 25 ans en recherche partenariale. Il est président du Réseau québécois en innovation sociale (RQIS). Toutefois, c'est son rôle de directeur général de l'organisme Territoires innovants en économie sociale et solidaire (TIESS) qui retient surtout notre attention dans le cadre de cet entretien.

-.Vincent van Schendel, merci de nous accueillir dans les bureaux de l'organisme Territoires innovants en économie sociale et solidaire pour un entretien sur l'innovation territoriale, plus précisément sur cet organisme dont tu es le directeur général.

Vincent van Schendel. J'ai toujours beaucoup de plaisir à discuter de ce sujet, et tout particulièrement de la mission et des réalisations du TIESS, dont je suis le directeur général depuis sa création en 2014. Cela me fait d'autant plus plaisir d'en parler avec Juan-Luis Klein, qui était présent dès le début.
Je crois que la création du TIESS, un organisme de liaison et de transfert en innovation sociale (OLTIS), a été toute une aventure... Peux-tu nous en rappeler l'histoire?

V. v S. Oui, ce fut toute une aventure! En fait, je suis impliqué au TIESS depuis au moins six ans et on pourrait même dire sept ans, soit l'année avant qu'on obtienne officiellement le financement du ministère du Développement, de l'Économie, de l'Innovation et des Exportations (MDEIE), devenu le ministère de l'Économie et de l'Innovation (MEI). En janvier 2012, on avait déposé une lettre d’intention au Ministère en réponse à un appel d'offres pour la création d'un OLTIS1. Et quand je dis « on », je réfère au Centre de recherche sur les innovations sociales 
(CRISES), au Chantier de l'économie sociale, à l'Institut Karl-Polanyi d'économie politique de l'Université Concordia et au Service aux collectivités de l'UQAM, où j'étais coordonnateur.

La lettre d'intention ayant été retenue, nous avons dû déposer tout le dossier, incluant 43 lettres d'appui, dès mars 2012. Puis, nous avons obtenu le financement. Juan-Luis Klein du CRISES et Nancy Neamtan du Chantier de l'économie sociale ont beaucoup travaillé auprès du Ministère pour convaincre les responsables du programme de la pertinence d'un tel OLTIS.

\section{D'où vient lidée du TIESS?}

V. v S. J'y vais un peu en vrac, au risque d'être décousu. En 2011, une demande de financement pour un projet plus large en innovation sociale avait été déposée au MEI. Celle-ci avait été refusée faute de fonds et parce que le projet avait été jugé trop large. On a alors décidé de déposer un projet plus ciblé en économie sociale. Forts de plus de 30 années de collaboration, les quatre organismes que je vous ai mentionnés ont monté le projet en trois mois. On a obtenu le financement et on a sauté de joie! La première chose qu'on a faite a été de parler de gouvernance et de mettre sur pied un conseil d'administration provisoire. On s'est dit qu'il faudrait nommer un directeur général. Tout le monde s'est regardé, puis Juan-Luis Klein et Nancy Neamtan, qui ont été co-présidents du TIESS de sa création jusqu'en 2018, m’ont demandé : " Ça te dirait? » Je leur ai répondu : «Non! (Rires) Vous ne voulez pas ça! D’abord, je ne suis pas un gestionnaire, et je suis toujours à la dernière minute. C'est l'enfer, travailler avec moi! »

\section{Manifestement, ils ont réussi à te convaincre. Comment ont-} ils fait?

V. v S. Ils m'ont promis de m'aider et j'ai décidé d'essayer. Je ne savais pas trop dans quoi je m'embarquais... Le TIESS n'est pas un truc de gestion; c'est plus un truc de vision. De mettre ensemble des perspectives et des acteurs pour transférer des connaissances, mais dans la pratique des organisations. Ça promettait d'être un débat fantastique sur des questions telles que: C'est quoi, transférer des connaissances? C'est quoi, des connaissances? Des pratiques? C'est quoi, transformer? C'est quoi, la mission du TIESS? On veut faire du développement territorial, mais c'est quoi, le développement territorial? On est en innovation sociale, alors c'est quoi, l'innovation sociale? On travaille avec des chercheurs, alors comment travaille-t-on avec des chercheurs? Avec des praticiens? Avec lesquels? Et il a fallu gérer des conflits. Or, on ne partait pas de rien parce qu'il y avait eu quand même 10 ans de travail au sein de l'Alliance de recherche universitéscommunauté en économie sociale (ARUC-ÉS).

Vous ne partiezpas de rien... Quels étaient vos expériences, vos compétences, vos acquis?

V. v S. Il y avait des connivences, des gens qui se connaissaient et des pratiques communes, mais plus dans le domaine de la recherche. Dès le début de l'ARUC-ÉS en 2000, Benoît Lévesque (actuellement professeur émérite du Département de sociologie de l'UQAM), alors directeur du CRISES et de l'ARUC-ÉS, avait fait une demande au MEI, avec le Chantier de l'économie sociale, pour avoir un centre de transfert. On se disait : «C'est bien beau la recherche, mais on veut aussi transférer ces connaissances dans des organisations. » Donc, la première tentative de faire un lieu de transfert a eu lieu en 2000. Puis, pendant 10 ans, on nous a répondu : "C'est bien intéressant, mais il n'y a pas d'argent pour ça. » À force d'insister, nous avons motivé le Ministère, qui a mis sur pied un concours, et c'est là qu'on a obtenu le financement. Bref, il y avait quand même 10 ans de pratiques communes à l'ARUC-ÉS.

\section{En fin de compte, quel est le mandat du TIESS?}

V.v S. Le mandat du TIESS est de faire du transfert de connaissances, pas de la recherche. Toutefois, on travaille avec des chercheurs et on avait ce noyau de connivences, de pratiques communes, de langage commun, de complicité et je dirais de vision sur l'innovation sociale, sur l'économie sociale et sur le développement territorial. Nous avons tout de même dû expliciter cette vision parce que, au sein du TIESS, il n'y avait pas seulement le CRISES, le Service aux collectivités, le Chantier de l'économie sociale et l'institut Karl Polanyi : il y avait aussi d'autres centres de recherche et d'autres réseaux comme le Conseil québécois de la coopération et de la mutualité (CQCM), les pôles d'économie sociale en région, une série de réseaux sectoriels... Ce qui fait qu'aujourd'hui, le TIESS regroupe 70 établissements, réseaux et centres de recherche. 
Quand des gens de l'étranger nous visitent, ils sont étonnés, alors que nous, on ne s'en rend pas compte au quotidien. Ces gens-là nous regardent avec de grands yeux: "Vous mettez ensemble 3 centres de recherche, 11 universités, 6 établissements secondaires, de grands réseaux... et ça fonctionne? » Ça fonctionne, mais il y a des hauts et des bas. Parfois, on ne se comprend pas. Donc, il a fallu aussi essayer de formaliser tous ces mécanismes de coconstruction. Parce qu'ils ne tombent pas du ciel...

Justement, parle-nous du fonctionnement du TIESS.

V. v S. Dans une telle structure, il faut réguler les rapports et construire. L'espace de construction est un espace en soi : il a ses propres règles. On met ensemble le milieu de la recherche et le milieu de la pratique. Chaque milieu a ses contraintes, son langage, ses objectifs. Or, il y a quand même un espace de collaboration qui obéit à ses propres règles. On a donc construit les règles de cet espace et les mécanismes de régulation. Mine de rien, notre équipe approche les 20 personnes, ce qui inclut l'équipe à Montréal et les personnes en région. On a plusieurs projets avec des outils et des fonctions qui se développent, une gestion qu'il faut développer, beaucoup de partenariats, un conseil d'administration de plus de 20 membres, un comité exécutif, différents mécanismes de travail et un conseil scientifique. On fait parfois des erreurs, mais on corrige le tir et on s'ajuste.

Malgré tout, il y a cette connivence qui se maintient depuis six ans et on arrive à construire des outils non seulement des outils très concrets et praticopratiques, mais aussi un dialogue permanent sur les grands enjeux, dont l'innovation sociale, le développement des territoires, la transition écologique et la démocratisation de l'économie. Finalement, une grande discussion se poursuit, d'un projet à l'autre et d'une instance à l'autre.

Quel est le rôle du TIESS en ce qui a trait au développement territorial?

V. v S. Quand TIESS a été fondé, c'était en 2013, juste avant le tsunami politique de 2014. Il y avait alors des corporations de développement économique communautaire (CDEC), des centres locaux de développement (CLD) et des conférences régionales des élus (CRÉ). Un an après, il n'y avait plus rien, ou presque. Ça nous a forcés à nous demander: Mais pourquoi fallait-il faire du développement territorial? Pourquoi en faut-il encore? À ce moment, plusieurs personnes se sont tournées vers nous en nous demandant d'assumer des fonctions de liaison à travers les territoires et d'organiser des événements pour que les gens puissent se parler. C'est ainsi qu'on a organisé notre premier événement à Nicolet, en 2015.

\section{Rappelle-nous ce qui est ressorti de ce premier événement.}

V. v S. Lors de ce premier événement, 250 participants sont venus de partout, de tous les réseaux du développement territorial. On a compris qu'on avait besoin d'une espèce de terrain neutre pour que les réseaux se parlent. On avait une capacité de mobilisation parce qu'on collaborait avec une multitude de réseaux différents qui, entre eux, ne se parlaient pas. Nous permettions de faire des liens avec différents chercheurs qui se connaissaient, mais qui ne travaillaient pas ensemble. Ça nous a donné un souffle imprévu!

Ça nous a amenés à jouer un rôle qu'on n'avait pas du tout vu venir, et qui est encore présent, même si on fait toujours attention parce que notre rôle est le transfert, et non la représentation politique. Il y a tout de même des zones grises : on a toujours un pied à la limite du développement, du politique et de la recherche. On ne doit faire ni l'un ni l'autre, mais le transfert se fait dans l'action et on est toujours un peu dans les trois zones. Reste que cet espace neutre qu'on était, qu'on est et qu'on veut toujours être permet de faire des débats qui autrement ne se faisaient plus.

L'organisme Solidarité rurale du Québec est disparu... Enfin, il existe encore formellement, mais plus sous la même forme et il n'y a plus de financement. Donc, on a aussi tissé des liens avec des milieux ruraux. On a discuté avec le MEI et des gens de différentes directions régionales, puis on a mis en relation des fonctionnaires qui ne se connaissaient pas. C'est notre fonction de liaison.

Le TIESS a trois fonctions : la liaison, la veille et le transfert. Comment structure-t-on? C'est un débat qu'on a encore maintenant. Comment travaille-t-on avec les régions? Comment fait-on de la veille? Comment fait-on des transferts? On en a encore pour un moment à se poser ces questions-là. 
Comment en es-tu arrivé à faire de la liaison, de la veille et du transfert en développement territorial?

V. v S. En 1987, au Service aux collectivités de l'UQAM, où je travaillais avant d'être à l'ARUC-ÉS, j'étais embauché pour faire un programme de formation économique et syndicale. Le Service aux collectivités, c'est un service de l'UQAM qui fait la liaison et qui structure des partenariats de formation et de recherche entre l'UQAM, donc des professeurs de l'UQAM, des syndicats ainsi que des groupes de femmes, écologiques et communautaires. Au début, l'économie sociale n'était pas mentionnée, mais ça s'est imposé progressivement. Il y avait - et il y a toujours - un protocole d'entente entre l'UQAM et les centrales syndicales qui définit ce programme de formation économique syndicale sur l'emploi, avec la collaboration des différents professeurs. J'ai été embauché comme coordonnateur de ce programme. Après deux ans, il était terminé.

On a déposé une autre version de ce programme, mais qui, cette fois, était plus axé sur le développement régional et local parce que le besoin identifié avec les centrales syndicales, c'était les régions. On a fait un programme de développement économique régional et, de là, on s'est dit : «Ce n'est pas seulement de l'économie; c'est du développement territorial. »

On était à la fin des années 1980, au début des années 1990. C'était au temps où les premières CDEC émergeaient à Montréal. Elles avaient des pratiques de développement territorial, de développement économique communautaire, de concertation et de développement autrement. On est donc allé voir les CDEC, puis on s'est dit qu'on pourrait élargir nos activités, qui devenaient de la recherche et de la formation pour le développement territorial et le développement économique communautaire. Cela nous a amenés à documenter différentes initiatives.

Par exemple, on a travaillé avec la Société de développement Angus (SDA), dans la région montréalaise, pour documenter le projet Angus, avant même que la SDA ne soit créée. On cherchait à savoir quels étaient les systèmes locaux de production, les expériences ailleurs et les besoins d'adaptation de la main-d'œuvre du quartier. Il y a eu différents projets Angus sur quelques années, avec plusieurs professeurs. Il y a donc eu tout un processus d'accompagnement qui est important parce que ça revient maintenant. Les collaborations qui sont apparues à ce moment sont en train de revenir... après une très longue parenthèse de près de 20 ans! Les collaborations sont en train de redémarrer sur le développement de l'Est de Montréal - j’y reviendrai. C'est là que se sont développées ces collaborations et, dans le très concret, que s'est forgée aussi toute une collaboration à partir de la pratique, mais aussi de l'expérience de chacun des professeurs.

En plus des bénéfices pour le quartier Angus, comment cette expérience a-t-elle influencé ta façon de voir le développement territorial?

V. v S. Il s'est construit une pensée voulant qu'on puisse faire autrement; on peut développer autrement et on peut travailler ensemble. On n'est pas obligés d'avoir des chercheurs qui cherchent et des développeurs qui développent. Il y a d'autres façons de faire qui sont en émergence. Le projet de ce qui allait devenir la SDA, c'était un projet complètement fou quand on y pense: redéployer un vaste terrain industriel sur le modèle des systèmes locaux de production pour créer des centaines d'emplois et redonner vie à un quartier entier. La CDEC Rosemont Petite-Patrie, puis la SDA, se sont associés à une équipe de chercheurs et, dans le cadre des services aux collectivités de l'UQAM, on a travaillé le concept pendant des années: des documents ont été produits, on a tenu un colloque international, on a publié un livre sur le sujet. Au début, on se réunissait à cinq dans un petit local à l'UQAM et on définissait un avenir possible. Bien sûr, il a fallu un porteur pour que ça réalise, mais ça a été un intense travail conjoint d'exploration et de définition pendant des années. Puis, le projet Angus est parti dans une phase de développement, que je qualifierais d'immobilière. C'était très « briques et béton et financement». Puis, la SDA grossissant, elle s'est impliquée dans le développement de l'Est de Montréal en général.

Aujourd'hui, on travaille de nouveau ensemble (TIESS, le CRISES et la SDA), mais aussi avec beaucoup d'autres organismes, dont la Chambre de commerce de l'Est de Montréal, l'Association industrielle de l'Est de Montréal et le CIUSSS de l'Est-de-l'Île-deMontréal, pour organiser un forum sur la relance de l'Est en dehors des pouvoirs publics. À travers ce projet, encore une fois, il y a 30 ans de réflexions, de travail et de pratiques sur les façons de développer des territoires autrement que seulement par des terrains industriels. 
Le TIESS a été appelé à prendre le relais des CDEC, des CLD et des CRE en créant des espaces de dialogue. Or, le TIESS ne fait pas de développement territorial comme tel. Qui remplace ces organismes? Comment le TIESS peut-il contribuer à ce que l'histoire ne se répète pas?

V. v S. À la suite de l'expérience des CDEC, les CLD ont été mis sur pied par le gouvernement du Québec. C'était en 1997, à la suite du Sommet sur l'économie et l'emploi de 1996. De 1997 à 2014, les CLD et les CDEC ont fait des choses intéressantes qui sont, d'une certaine façon, disparues. Là aussi, il y a tout un débat qui n'est pas terminé. Comment ont-ils pu disparaitre si facilement? Peut-être justement parce qu'on n'avait pas systématisé l'expérience du développement local, que chacun des CLD travaillait de façon différente en vase clos, qu'il n'y avait pas de pensée ni de vision commune.

À travers ces expériences et ces échecs s'est construite la compréhension que le transfert des connaissances, c'est important pour le développement territorial. Ce n'est pas seulement une histoire de faire des recherches et de les diffuser. C'est comment on se donne une vision commune, et comment la recherche et l'expérimentation sont aussi des moteurs de l'action sur le terrain.

La vision et la compréhension qu'on en a, c'est majeur pour fonctionner, développer, faire des alliances, expliquer, mobiliser. Une des leçons à tirer de l'abolition des CDEC et des CLD, c'est que le développement territorial va continuer. Il va continuer sous d'autres formes. En fait, c'est le même principe, mais porté par d'autres organisations.

Ça prend du temps, c'est en train de se reconstruire, mais c'est important d'intégrer désormais une dimension de transfert dans le développement, de reprendre l'approche innovatrice, de reconstruire ces innovations sociales que constituaient les processus démocratiques, les gouvernances locales, les nouveaux montages financiers et l'hybridation des ressources. Il y a aussi des apprentissages qui viennent de l'économie sociale dans sa façon de bénéficier d'une hybridation des ressources et des approches. Alors, cette compréhension du transfert s'est construite progressivement comme intégration dans la pratique des organisations.

Le transfert, ce n'est pas seulement transférer les connaissances de la recherche vers le terrain; c'est un processus bilatéral - même multilatéral - d'échange, de partage de connaissances. C'est essentiel au développement. Le transfert est une fonction à part entière, mais ce n'est pas une fonction technique. C'est une fonction qui, elle-même, implique une mixité de collaborations, autant sur le terrain que dans le milieu de la recherche. Le transfert, c'est ce que le TIESS veut favoriser pour que les choses se fassent différemment. Et c'est un métier en soi, mais c'est un métier qu'on développe.

\section{Justement, parle-nous de ce métier.}

V.v S. On appelle les employés du TIESS des conseillères en transfert. Au début, on a eu un débat sur l'appellation de cette fonction parce que ça n'existe nulle part. Le rôle du conseiller en transfert, ce n'est pas seulement de l'intermédiation, ni un courtier, ni une mise en relation. C'est une traduction. Oui, c'est une relation, mais ce n'est pas : «Je te présente telle personne, puis arrangez-vous.» C'est plutôt une liaison : «Je pense que vous avez de quoi vous entendre; on va s'entendre sur quelque chose de concret. » On apprend à travailler ensemble dans des univers différents avec des langages différents. Pourtant, on travaille ensemble, puis on construit ensemble et ça répond aux aspirations de chacun. Et ça, c'est un métier.

Un métier qu'on n'apprend pas encore dans les universités - un jour, peut-être - et qui, je crois, devrait aussi faire partie de la formation dans les futurs métiers de développement territorial. Parce que le transfert est une composante du développement. Donc, il faut être capable de mettre en relation. Quand on fait du développement territorial, on met en relation les gens, on crée des consensus, on gère des conflits. À travers cette gestion et cette régulation de conflits, on arrive à dégager des perspectives qui nous permettent d'avancer.

Le transfert implique tout ceci, mais aussi de transférer les connaissances pour éviter de recommencer à apprendre sur le tas pendant trois ans avant d'avancer. Peut-on faire le bilan des connaissances qui existent? Peut-on repérer les initiatives en travail de veille? Peut-on mettre ensemble les gens pertinents, le travail de mise en relation, de liaison? Ainsi, on voit comment l'expérience académique ou pratique des uns et des autres peut être reprise par d'autres.

On peut faire une analogie avec la botanique. Disons qu'on veut transplanter des tulipes. On va 
aller chercher des bulbes dans la terre, puis on va les planter dans l'autre terre à côté. Donc, on transfère les bulbes dans une autre terre. Ce n'est pas la même composition de sol, ni la même exposition au soleil, ni le même vent, donc les tulipes ne pousseront pas de la même façon. Le transfert n'implique pas la réplication à l'identique; c'est aussi une adaptation. C'est comment on intègre dans sa pratique les connaissances issues de la pratique des autres. Or, en plus d'impliquer une adaptation, ça implique d'abord une systématisation.

Par exemple, on a fait une expérience à un endroit. On peut faire comme en cuisine : on a des oignons, des carottes, de la farine, du lait, du bœuf, et je ne sais quoi encore. Ça ne fait pas un repas : il faut cuisiner les ingrédients avec une recette, puis les mettre au four à la bonne température, le temps qu'il faut. Ça prend des cuisiniers pour que ce soit mangeable - et même bon. Alors, il y a beaucoup de points communs entre le développement territorial et le transfert. Le conseiller en transfert est un métier de cuisinier, de mise en récit.

Outre ce métier passionnant, il y a l'aventure du TIESS elle-même qui est passionnante parce qu'on fait des choses qui n'existaient pas avant, mais, en même temps, on s'enracine dans plein de choses qui existent.

Pour faire les liens entre les personnes et transférer des connaissances, il faut connaître les personnes et avoir les connaissances. Comment cela se fait-il? Que font les conseillers et conseillères en transfert au quotidien?

V.v S. Une bonne partie de notre travail consiste à faire le tour des expériences et connaissances actuelles pour être sûrs qu'on ne refasse pas les mêmes faux pas. C'est ce qu'on appelle des synthèses de connaissances, qui sont autant théoriques que pratiques. Ce n'est pas juste une revue de littérature. Souvent, il faut aller voir sur le terrain pour savoir ce qui a été fait et faire des entrevues. En fait, c'est de se demander : Qu'est-ce qu'on sait? Qu'estce qu'on sait qu'on sait? Qu'est-ce qu'on sait qu'on ne sait pas? Et, parfois, qu'est-ce qu'on ne sait pas qu'on sait? Parce que ce qu'on sait, ce sont des savoirs tacites. Il y a beaucoup de choses qu'on sait... mais on ne le sait pas! Michael Polanyi, dans son essai intitulé Tacit Knowledge, a écrit : «Les gens ne savent pas toujours ce qu'ils savent. » Donc, il faut aussi savoir ce qu'on sait et le valoriser. Parce qu'il y a des connaissances immenses dans les pratiques existantes, mais qui ne sont pas vues, connues, reconnues ni valorisées.

Ici, on pourrait ouvrir toute une parenthèse sur la connaissance, sur le savoir. Dans mon temps hors travail, je siège aussi au conseil d'administration de l'organisme Paroles d'excluEs, qui a développé toute une approche de croisement des savoirs. Cet organisme s'est beaucoup inspiré des travaux d'ATD Quart Monde, en France, qui a publié un livre intitulé Croisement des savoirs. En résumé, il y aurait trois types de savoirs : le savoir académique, le savoir d'expérience et le savoir vécu.

Le savoir vécu, c'est par exemple quelqu'un qui habitait un pays chaud, qui arrive au Québec et qui sort en sandales en janvier. Il apprend vite à enfiler des bottes chaudes, sans l'avoir lu nulle part ou sans que personne le lui ait expliqué. Seulement grâce à son expérience de vie, il comprend qu'il ne faut pas faire ça parce que sinon il gèle... Il le vit et il l'apprend. Il y a aussi le savoir académique qui est franchement plus valorisé, plus développé. Il est institutionnalisé: dans les institutions, dans des revues, dans des cours, dans un langage, dans des événements. Donc, croiser le savoir académique avec un savoir d'expérience de groupes ou de personnes marginalisées ou encore avec le savoir vécu, ce n'est pas égal. La négociation ne peut pas se faire toute seule.

Ainsi, il y a aussi un rapport de pouvoir dans le rapport des savoirs. Ça prend une médiation organisée. Transférer des connaissances, ça implique de les valoriser. Donc, si on veut les valoriser, il faut les reconnaittre et leur permettre de s'exprimer. Pour le bien de tout le monde d'ailleurs, parce qu'il y a aussi les connaissances très terrain qui peuvent être reprises à l'université.

Donc, un de nos objectifs est que le transfert se fasse dans les deux sens et que ça puisse alimenter les pratiques d'enseignement, les types de recherche, les façons de faire de la recherche, les façons de capter des connaissances. Or, l'idée pour nous est toujours de transférer l'ensemble de ces connaissances, une fois traduites, mélangées et cuisinées, mais dans la pratique. 
Au-delà d'accumuler des connaissances, peux-tu nous dire comment cela contribue au développement des territoires et permet l'innovation sociale? Comment cela sert-il vraiment? Quel en est l'impact?

V. v S. L'objectif est que ça serve à transformer. Ce n'est pas une transconnaissance dans l'air: Pourquoi travaille-t-on sur un sujet ou un autre? sur les obligations communautaires? sur l'alimentation? sur la relance de l'Est de Montréal? Parce qu'il y a des actions en cours et des acteurs mobilisés. Donc, l'idée est de transférer dans cette pratique, dans l'Est de Montréal... Et transférer, ça peut prendre différentes formes.

Par exemple, c'est quand les chercheurs disent: «Écoutez, il y a eu diverses expériences de relance à travers le monde, ici et là. Il y a eu Boston, Barcelone, Lyon... Voici comment ils ont fait. » On peut déjà s'en inspirer, mais il faut approfondir, entre autres avec des pédagogies. Avec des chercheurs du CRISES, on a travaillé auprès de la municipalité de Saint-Camille dans le cadre d'un projet intitulé Les Ateliers des savoirs partagés. L'un des leaders de cette communauté disait: "Il y a une pédagogie de la visite. Quand on rend visite ou qu'on reçoit, on se présente. Et là, il y a quelque chose qui se passe dont on sort transformé. De la rencontre, on sort transformé. Après, notre pratique ne sera plus la même. »

Alors, la rencontre, ça peut être une visite, un comité de suivi, une instance. La chimie qui se passe dans une rencontre fait que la perspective de chacun est appelée à évoluer. Bien sûr, lors d'une courte visite de deux heures, l'impact peut être limité. Quand ce sont des visites qui se répètent sur plusieurs années, dans des mécanismes plu réguliers, le pari qu'on fait - et ce qu'on constate aussi -, c'est que les pratiques des uns et des autres se transforment. On apprend aussi à réfléchir ensemble, même si c'est de façon différente.

Alors, comment arrive-t-on à transformer des pratiques concrètes? Par exemple, on se demande comment des organisations peuvent émettre des obligations communautaires pour se financer. On a accompagné le Bâtiment 7 à Montréal, qui a émis des obligations pour se financer. Ça n'assure pas l'avenir du Bâtiment 7 puisqu'il y a plusieurs enjeux de financement, mais ça a permis de le financer en partie. On lui vient en soutien parce qu'on a transféré l'expérience des autres dans sa pratique. Ça peut être très concret.
Ce sont des enjeux très spécifiques auxquels font face des oryanisations on des milieux précis. Est-ce que le TIESS s'intéresse à des enjeux plus globaux de développement territorial?

V. v S. Oui. Il y a aussi des questions plus larges comme celle du modèle de développement actuel, qui nous mène vers la catastrophe. Il faut donc mettre en place une transition écologique. Ça veut dire qu'il faut réfléchir ensemble, tous les réseaux terrain et tous les chercheurs et les établissements, et se demander: Comment peut-on transformer ou même arrêter le train dans lequel on est? Parce qu'on doit le faire tourner, sinon changer de train. Il y a une urgence. On veut transformer l'état des choses. Le TIESS devient alors un outil dans une volonté plus globale de transformation.

C'est là que l'innovation et le développement territorial reviennent en force parce qu'un des éléments centraux pour une transformation, c'est qu'elle soit ancrée dans le territoire; qu'on redéveloppe des circuits courts; qu'on favorise l'autonomie, la propriété collective, donc les petites unités - mais il peut aussi y avoir de plus grandes unités, car ce n'est pas forcément l'apologie du petit. L'économie sociale est aussi appelée à changer d'échelle.

C'est donc de s'interroger sur la façon de favoriser l'essaimage : il y a des expériences ici qui peuvent profiter à d'autres ailleurs, ce qui peut transformer progressivement le modèle de développement. On le voit dans les secteurs de la mobilité et de l'alimentation, où le TIESS devient un outil dans une volonté de transformation, mais avec toujours cet impératif de systématiser.

Il existe des centaines et des milliers d'expériences d'alimentation qui souvent vivotent, qui se cassent la gueule. Or, à travers ces expériences, il y a des apprentissages incroyables qu'on peut tirer, qui peuvent profiter à d'autres. Actuellement, il y a un besoin d'apprendre les uns des autres; que tout le monde se parle. On est en train de monter un projet, puis on a les moyens de le faire parce qu'encore une fois, c'est cet apprentissage mutuel qui nous permettra de développer autrement dans l'alimentation. Et c'est pareil dans la culture, dans le numérique collectif, dans l'immobilier, etc. C'est vraiment le territoire comme lieu d'ancrage des initiatives qui devient majeur.

On revient donc encore au développement territorial, et ce sont de nouvelles façons de faire, de gérer, 
de développer, d'être propriétaire, de financer. C'est ça, l'innovation sociale. Elle peut d'ailleurs être très liée à une revitalisation, mais comme processus collectif; on parle alors d'innovation collective. Actuellement, dans le mouvement de l'entrepreneuriat social, souvent, on voit plutôt l'innovation comme une idée individuelle qu'on veut développer.

Il y a même maintenant un autre courant en innovation sociale, entre autres dans les sociétés de valorisation. Il y a un chercheur qui développe une idée, qui la commercialise et qui la vend comme de l'innovation sociale. C'est le même mot, mais ce n'est pas du tout la même chose! Il y a donc un enjeu sémantique. Il faut clarifier ce terme et les concepts sous-jacents. Derrière le même mot, on parle de choses très différentes. Pour nous, c'est clair qu'on est dans un processus collectif, dans une volonté de transformer le modèle de développement, d'aller vers une transition écologique. Ce qu'on fait, finalement, c'est viser à outiller les mouvements qui sont dans l'action, dans cette visée d'innovation sociale. $\bullet . \bullet$

\section{Nous remercions Monsieur Vincent van Schendel pour cet entretien fort intéressant!}

\section{NOTES}

1 Les organismes de liaison et de transfert en innovation sociale (OLTIS) ont été créés dans le cadre de la Stratégie québécoise de la recherche et de l'innovation adoptée en 2007 par le ministère du Développement, de l'Économie, de l'Innovation et des Exportations (MDEIE). Il était prévu de créer trois OLTIS et deux ont été fondés. Puis, en 2012, il y a eu un appel de projets pour un troisième OLTIS. 